Provided for non-commercial research and education use. Not for reproduction, distribution or commercial use.

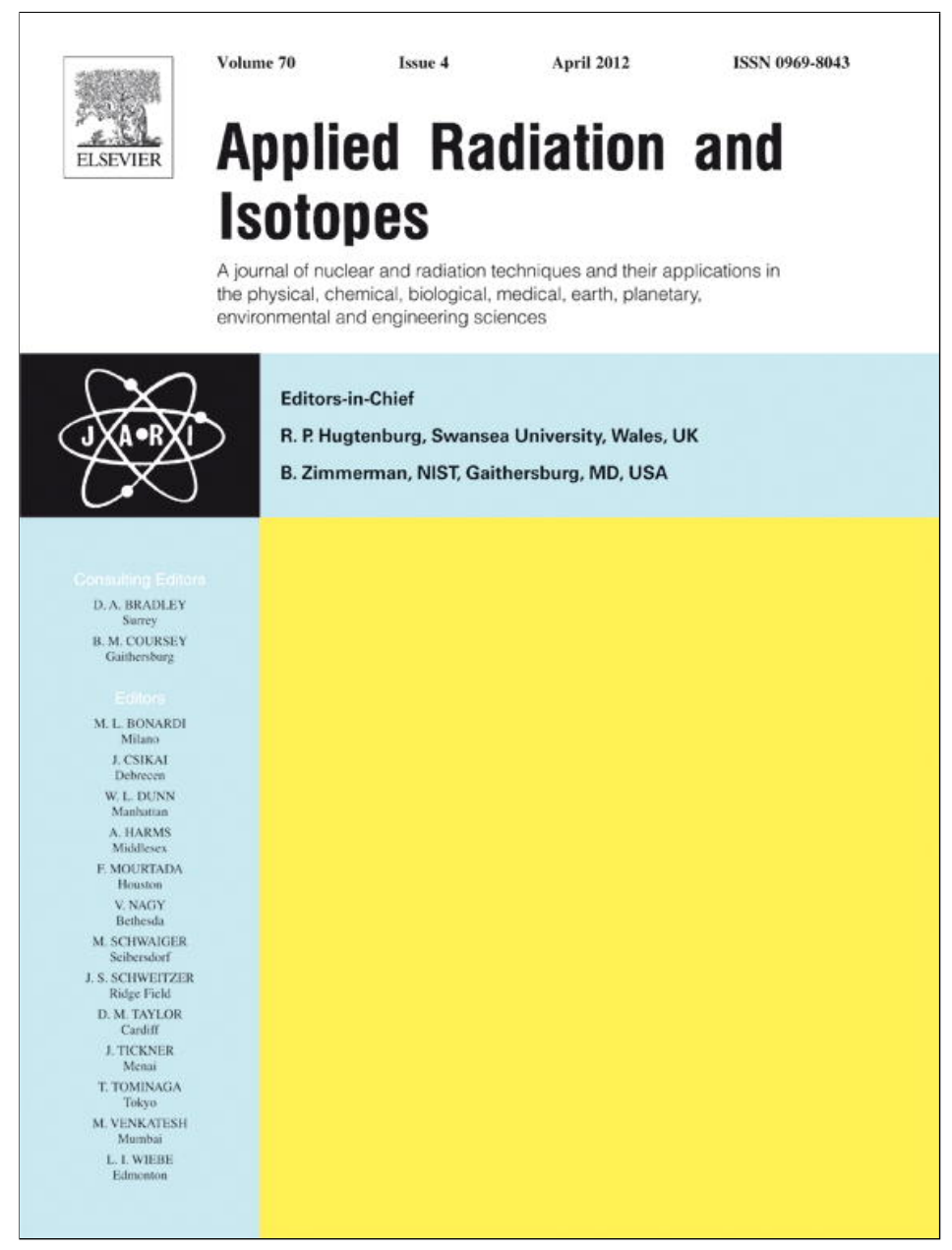

This article appeared in a journal published by Elsevier. The attached copy is furnished to the author for internal non-commercial research and education use, including for instruction at the authors institution and sharing with colleagues.

Other uses, including reproduction and distribution, or selling or licensing copies, or posting to personal, institutional or third party websites are prohibited.

In most cases authors are permitted to post their version of the article (e.g. in Word or Tex form) to their personal website or institutional repository. Authors requiring further information regarding Elsevier's archiving and manuscript policies are encouraged to visit:

http://www.elsevier.com/copyright 


\title{
The implications of particle energy and acidic media on gross alpha and gross beta determination using liquid scintillation
}

\author{
D. Zapata-García, M. Llauradó*, G. Rauret \\ Laboratori de Radiologia Ambiental (LRA), Departament de Química Analítica, Universitat de Barcelona, Martí i Franqués, 1-11 Planta 3, E-08028 Barcelona, Spain
}

\section{A R T I C L E I N F O}

\section{Article history:}

Received 10 January 2011

Received in revised form

24 November 2011

Accepted 8 December 2011

Available online 16 December 2011

\section{Keywords:}

Liquid scintillation

Alpha/beta discrimination

Alpha/beta calibration

Gross alpha/beta measurement

Drinking water

\begin{abstract}
A B S T R A C T
The interaction of humans with radioactivity present in the environment from natural and artificial sources necessitates an evaluation of its risk on human health. Gross alpha and gross beta activities can provide a rapid evaluation of the radioactive content of a sample and can be simultaneously determined by using liquid scintillation counters. However, calibration of the liquid scintillation counter is required and is affected by many factors, such as particle energy and the acidity of the media. This study investigates what effect the particle energy used for calibration has on misclassification and how to account for this misclassification in routine measurements.

The variability in measurement produced by the final $\mathrm{pH}$, as well as any acids used in sample treatment, was also studied. These results showed that the most commonly used acid for these types of analyses, $\mathrm{HNO}_{3}$, produced a high amount of misclassifications at very low $\mathrm{pH}$. The results improved when $\mathrm{HCl}$ was used to adjust the sample to low $\mathrm{pH}$.
\end{abstract}

(c) 2011 Elsevier Ltd. All rights reserved.

\section{Introduction}

The presence of radioactivity in the environment is caused mainly by naturally occurring radionuclides and cosmic radiation, although there is also a contribution from artificial sources. Unnatural sources include the fallout from nuclear tests and accidents and human activities, such as nuclear power plants and NORM industries.

Radionuclides are present in all aspects of the environment, soils, water, biota and air, and the continuous interactions of humans with the environment makes it necessary to evaluate the risk of radionuclides on human health.

Radioactivity in water can reach humans and the environment through many different mechanisms because it is a resource that is directly consumed, used in food processing and used in many industries (Mas et al., 2007). Many countries have drinking water regulations (European Council, 1998; USEPA, 2000), although according to the World Health Organisation (2008) the exposure to radioactivity through water is small.

Radioactivity monitoring requires adequate representative parameters that can be easily determined and simple methods that are easy to apply to a wide range of samples. For many applications, the determination of gross alpha and gross beta activity provides a rapid evaluation of the radioactive content of

\footnotetext{
*Corresponding author. Tel.: +34934029 083; fax: + 34934021233.

E-mail address: montse.llaurado@ub.edu (M. Llauradó).
}

a sample and can be used to decide whether specific radionuclide determinations requiring longer and more expensive analyses are necessary. According to World Health Organisation guidelines (2008), water is considered adequate for human consumption when the gross alpha activity concentration is below $0.5 \mathrm{~Bq} \mathrm{l}^{-1}$ and the gross beta activity concentration is below $1 \mathrm{~Bq}^{-1}$. When the values are higher, the determination of specific radionuclides should be carried out. Countries may have different threshold values for these two parameters, as in the case of Spain, where the limit is $0.1 \mathrm{~Bq}^{-1}$ for gross alpha activity and $1 \mathrm{~Bq} \mathrm{l}^{-1}$ for gross beta activity (Spain, 2003).

Liquid scintillation counting is an adequate technique for these analyses, due to the existence of ultra-low level detectors equipped with pulse-shape discrimination devices that allow the simultaneous determination of alpha and beta emitters. However, simultaneous determination requires calibration of the equipment to establish an adequate value for the pulse-shape discrimination parameter. This calibration is performed by individual measurement of the misclassification produced by pure alpha and pure beta emitters in order to find the point where the sum of both misclassification values achieves its minimum. The two main factors affecting calibration are quenching produced by the matrix and the energies of the radionuclides chosen. Since the sample is mixed with the scintillation cocktail, any substance present in the sample might interfere with the scintillation and other light emission processes. This effect has been studied using diverse chemical agents (DeVol et al., 2007; Palomo et al., 2011; Pujol and Sanchez-Cabeza, 1997; Rodríguez Barquero and Grau Carles, 1998; Villa et al., 2003), and Pates et al. 
(1998) proved that the quenching mechanism depends on the agent used. An alternative to quenching curves is to develop methods that guarantee the quenching values will be constant, for example, by acidifying the sample to a constant $\mathrm{pH}$ value, which simplifies the quantification and uncertainty reporting (Rusconi et al., 2006; Zapata-García et al., 2009).

The energies of the emitters used for the calibration also affect the response of the equipment. Many studies assumed that only beta energies should be considered because the beta energy range is much wider than the alpha energy range (Pates et al., 1998; Yang, 1996). More recent work provided evidence that alpha energies also influence the alpha/beta misclassifications (Salonen, $2006 \mathrm{~b})$. The most common radionuclides for calibration are ${ }^{241} \mathrm{Am}$ and ${ }^{90} \mathrm{Sr} /{ }^{90} \mathrm{Y}$. However, some authors use other alpha radionuclides, such as ${ }^{230} \mathrm{Th},{ }^{226} \mathrm{Ra}$, natural $\mathrm{U}$ and ${ }^{210} \mathrm{~Pb}$ and other beta radionuclides such as ${ }^{137} \mathrm{Cs},{ }^{40} \mathrm{~K},{ }^{32} \mathrm{P}$ or ${ }^{36} \mathrm{Cl}$ (Forte et al., 2007; Pujol and Sanchez-Cabeza, 1997; Salonen, 2006a; Wong et al., 2005). In a previous paper (Zapata-García and Llauradó, 2009), the performance of the Laboratori de Radiologia Ambiental (Environmental Radiology Laboratory, LRA) method was applied to synthetic and real samples. Despite good validation results, the method showed problems when testing water with high natural radioactivity content. This deficiency was probably due to the difference between the emission energies of the natural alpha emitters and the ${ }^{241} \mathrm{Am}$ used for calibration. One of the objectives of this paper is to study the effect that alpha energy has on misclassification and how this should be accounted for in routine methods where the energies of the emitters in a sample are unknown.

Many methods can be found in the literature that use liquid scintillation for gross alpha/beta determination. Most of them apply a concentration process to the acidified sample before mixing it with the scintillation cocktail (Dávila Rangel et al., 2001; Kleinschmidt, 2004; Ruberu et al., 2008). Concentration eliminates radon and its short-lived daughter isotopes and improves the minimum detectable activity (MDA). Validation of such methodologies is accomplished by analysis of synthetic samples and participation in intercomparison exercises. However, little has been published on the internal variability of these methods.

In this work, the variability produced by $\mathrm{pH}$ and the acid used in the treatment of samples was studied. Synthetic samples were analysed by applying the optimised conditions in order to evaluate how any remaining misclassification affected the results when the alpha and beta emitter levels were different

\section{Materials and methods}

\subsection{Instrumentation}

For pH measurements, a Cyberscan pH1100 (Eutech Instruments, Singapore) pH-meter was used. Gross alpha and gross beta activities were measured using a 1220 Quantulus (Wallac, Turku, Finland) ultra low-level analyser, which had a pulse shape discrimination device (pulse shape analyser (PSA)) and an external standard of ${ }^{152} \mathrm{Eu}$ for the measurement of external quench parameters (SQP[E]). Twenty-millilitre polyethylene vials (Perkin Elmer, Waltham, Massachusetts, USA) and an Ultima Gold AB scintillation cocktail (Perkin Elmer) were used.

All vials were maintained inside the counter for at least two hours to allow dark adaptation prior to counting. Standards were measured for 100 minutes and samples for 400 minutes.

Spectra were analysed using EASY View Spectrum Analysis Software. The counting windows were set to channels 550-800 in the alpha spectrum and channels $250-1024$ in the beta spectrum. Windows were chosen so that all alpha events in the $4-8 \mathrm{MeV}$ range and all beta events excluding ${ }^{3} \mathrm{H}$ could be detected.

\subsection{Reagents and solutions}

Double-deionised water was obtained from a Millipore water purification system, and analytical grade reagents were used throughout this study.

Commercial solutions of ${ }^{236} \mathrm{U}$ (Eckert \& Ziegler, Valencia, California, USA), ${ }^{241} \mathrm{Am}$ (Amersham, Amersham, Buckinghamshire, UK), ${ }^{90} \mathrm{Sr} /{ }^{90} \mathrm{Y}$ (Amersham) and ${ }^{137} \mathrm{Cs}$ (CERCA LEA, Pierrelatte Cedex, France) were used. A ${ }^{40} \mathrm{~K}$ standard was prepared by dissolving $\mathrm{KCl}$ (MERCK, 99.5\% pure) in water until saturation was reached. The ${ }^{40} \mathrm{~K}$ activity was then measured using high-resolution gamma spectrometry (Canberra BE 3830-7500SL, resolution 1.73). A ${ }^{90} \mathrm{Sr}$-free ${ }^{90} \mathrm{Y}$ standard was prepared by precipitating $\mathrm{Y}(\mathrm{OH})_{3}$ with $\mathrm{NH}_{4} \mathrm{OH}$ after the addition of a $\mathrm{Y}_{2} \mathrm{O}_{3}$ carrier. $\mathrm{Y}(\mathrm{OH})_{3}$ was dissolved using $\mathrm{HCl}(50 \%, \mathrm{v} / \mathrm{v})$.

\subsection{Samples}

A total of 20 synthetic samples were prepared for analysis using the different procedures. Solutions were prepared using ${ }^{236} \mathrm{U}$ and ${ }^{90} \mathrm{Sr} /{ }^{90} \mathrm{Y}$ as alpha and beta emitters, respectively, at three levels of activity concentration: slightly over the MDA and at concentrations approximately 1 and 2 orders of magnitude over MDA. The alpha and beta activity concentrations for the different samples are shown in Table 1.

A series of real samples with different levels of natural and artificial radionuclides were analysed in the final part of the study for method validation.

\subsection{Calibration}

The optimum PSA was established by calculating alpha and beta misclassification at different PSA settings. The effect of the beta energy was studied using 3 different beta emitters $\left({ }^{137} \mathrm{Cs},{ }^{40} \mathrm{~K}\right.$ and ${ }^{90} \mathrm{Y}$ ). The effect of the alpha energy was studied using 2 different alpha emitters $\left({ }^{241} \mathrm{Am}\right.$ and $\left.{ }^{236} \mathrm{U}\right)$. Once the PSA was established, the evaluations of the misclassification and the

Table 1

The gross alpha and gross beta activity concentrations of the synthetic samples used in the study. Concentrations are expressed in Bq $1^{-1}$.

\begin{tabular}{|c|c|c|c|c|c|c|c|c|}
\hline Sample & Alpha & Beta & Sample & Alpha & Beta & Sample & Alpha & Beta \\
\hline $\mathrm{A} 1$ & $4.78 \mathrm{E}-02$ & - & $\mathrm{AB} 1$ & $5.98 \mathrm{E}-02$ & 2.03E-01 & $\mathrm{AB} 1^{*}$ & $9.93 \mathrm{E}-02$ & $7.82 \mathrm{E}-01$ \\
\hline A2 & $4.80 \mathrm{E}-01$ & - & $\mathrm{AB} 2$ & $5.69 \mathrm{E}-02$ & $2.10 \mathrm{E}+00$ & $\mathrm{AB} 2 *$ & $9.98 \mathrm{E}-02$ & $2.43 \mathrm{E}+00$ \\
\hline A3 & $5.05 \mathrm{E}+00$ & - & AB3 & 5.69E-02 & $2.10 \mathrm{E}+01$ & $\mathrm{AB}^{*}$ & $9.80 \mathrm{E}-02$ & $2.37 \mathrm{E}+01$ \\
\hline B1 & - & $4.42 \mathrm{E}-01$ & AB4 & $5.61 \mathrm{E}-01$ & $2.10 \mathrm{E}-01$ & $\mathrm{AB} 4^{*}$ & $5.00 \mathrm{E}-01$ & 7.84E-01 \\
\hline B2 & - & $8.59 \mathrm{E}-01$ & AB5 & $5.60 \mathrm{E}-01$ & $2.08 \mathrm{E}+00$ & $\mathrm{AB5}^{*}$ & $4.98 \mathrm{E}-01$ & $1.94 \mathrm{E}+00$ \\
\hline \multirow[t]{2}{*}{ B3 } & - & $1.70 \mathrm{E}+01$ & AB6 & $5.41 \mathrm{E}+00$ & $2.05 \mathrm{E}-01$ & $\mathrm{AB} 6^{*}$ & $3.08 \mathrm{E}+00$ & 8.03E-01 \\
\hline & & & AB7 & $4.99 \mathrm{E}+00$ & $1.69 \mathrm{E}+01$ & $\mathrm{AB} 7^{*}$ & $3.00 \mathrm{E}+00$ & $2.34 \mathrm{E}+01$ \\
\hline
\end{tabular}


efficiency were accomplished using ${ }^{90} \mathrm{Sr}$ in secular equilibrium with ${ }^{90} \mathrm{Y}$ as beta emitter and ${ }^{236} \mathrm{U}$ as alpha emitter.

After the standard solutions were diluted to $8 \mathrm{ml}$ with water in the measurement vial, $12 \mathrm{ml}$ of Ultima Gold $\mathrm{AB}$ added, and the resulting mixture was shaken for 1 minute. When the $\mathrm{pH}$ was controlled, the water $\mathrm{pH}$ was adjusted with $2 \mathrm{M} \mathrm{HNO}_{3}$ or $2 \mathrm{M} \mathrm{HCl}$ solution before dilution of the standard. No $\mathrm{pH}$ variation was attributed to the standard addition because only a small amount of it was required $(<500 \mu \mathrm{g})$.

\subsection{Sample analysis}

Three different procedures were considered for the analysis of the synthetic samples. In Procedure $1,100 \mathrm{ml}$ of sample was acidified to $\mathrm{pH} 2.5 \pm 0.5$ with $\mathrm{HNO}_{3}$ and concentrated to $10 \mathrm{ml}$ on a hot plate with vigorous stirring in order to eliminate radon and its short-lived daughter isotopes. For Procedures 2 and 3, $100 \mathrm{ml}$ of sample was acidified with $\mathrm{HNO}_{3}$ to a final acid concentration of $0.5 \%(\mathrm{pH}<1)$ and evaporated to dryness on a hot plate. The final residue was diluted in $10 \mathrm{ml}$ of $\mathrm{HNO}_{3}$ at $\mathrm{pH} 2.5 \pm 0.2$ in the case of Procedure 2 and $\mathrm{HCl}$ at $\mathrm{pH} 1.5 \pm 0.2$ in the case of Procedure 3 . Measurement vials were prepared by mixing $8 \mathrm{ml}$ of the final solution with $12 \mathrm{ml}$ of Ultima Gold $\mathrm{AB}$ and shaking the mixture for 1 minute. For all three procedures, the sample treatment operations and vial preparation were controlled by weighing.

The detection limits were calculated using Currie's expression (1968) from the measurement of 10 blanks. MDAs were calculated after considering the efficiency, the counting time and the amount of sample. Blanks were analysed by applying the three procedures to $100 \mathrm{ml}$ of double-deionised water.

\section{Results and discussion}

\subsection{Calibration}

The curves of alpha and beta misclassification for the different emitters examined at different PSA values are shown in Fig. 1. Beta misclassification decreased as the PSA value increased. As can be seen in the figure, the percentage of misclassification in the case of a low-energy emitter, such as ${ }^{137} \mathrm{Cs}\left(E_{\max }=0.512 \mathrm{MeV}\right)$, is lower than for more energetic beta emitters. However, the expected increase in misclassification with increasing energies of the beta emitter was not observed for higher energies (the misclassification values for ${ }^{40} \mathrm{~K}\left(E_{\max }=1.312 \mathrm{MeV}\right)$ were higher than for $\left.{ }^{90} \mathrm{Y}\left(E_{\max }=2.284 \mathrm{MeV}\right)\right)$. This was so because while misclassified signals in the alpha multi-channel increased as the beta energy increased, for beta particles over a certain energy, misclassification took place in channel windows over the upper limit of the alpha window. In other words, part of the misclassified signal was not quantified, which causes the observed deviation.

In the case of alpha misclassification, the difference in the emission energies of the emitters caused the curve for ${ }^{236} \mathrm{U}$ $(4.494 \mathrm{MeV})$ to increase over $5 \%$ at PSA 107 , while for ${ }^{241} \mathrm{Am}$ $(5.485 \mathrm{MeV})$, this value was reached at PSA 145 . The most common natural radionuclides present in water are natural uranium $\left({ }^{238} \mathrm{U}, 4.196 \mathrm{MeV} ;{ }^{234} \mathrm{U}, 4.776 \mathrm{MeV} ;{ }^{235} \mathrm{U}, 4.396 \mathrm{MeV}\right)$ and ${ }^{226} \mathrm{Ra}(4.784 \mathrm{MeV})$. According to these results, calibration using ${ }^{241} \mathrm{Am}$ could induce errors in the analysis of water samples containing such radionuclides. In accident situations where high energy radionuclides, such as ${ }^{241} \mathrm{Am}$ or ${ }^{238} \mathrm{Pu}(5.499 \mathrm{MeV})$ would be found, calibration using ${ }^{236} \mathrm{U}$ would not cause problems; therefore, the final PSA value for further measurements was established at 110 according to the ${ }^{236} \mathrm{U}$ curve.

Misclassification and efficiency values were evaluated from the measurement of ${ }^{236} \mathrm{U}$ and ${ }^{90} \mathrm{Sr} /{ }^{90} \mathrm{Y}$ at the established PSA value. ${ }^{90} \mathrm{Sr}$ $\left(E_{\max }=0.546 \mathrm{MeV}\right)$ and ${ }^{90} \mathrm{Y}$ were used as intermediate- and highenergy beta emitters, respectively. ${ }^{40} \mathrm{~K}$ is the most common natural beta emitter and has an emission energy between ${ }^{90} \mathrm{Sr}$ and ${ }^{90} \mathrm{Y}$. The efficiency values were 95\% for gross alpha emission and $87 \%$ for gross beta emission, while the misclassification values were $6.3 \%$ (alpha) and 2.7\% (beta). ISO 11740 (2010) established a guidance value of $5 \%$ as the maximum interference in calculating sample activity. According to this guideline, these misclassification values are considered low enough that they were not taken into account during the quantification of samples.

\subsection{Evaluation of the established method's performance}

Procedure 1 (section 2.5) was established in the LRA for routine gross alpha/beta determinations. This procedure follows a commonly applied scheme consisting of preconcentration of water at controlled $\mathrm{pH}$ and further mixing with an adequate scintillation cocktail before measurement.

An evaluation of the error in the determination of gross alpha/ beta activity concentrations was carried out at three different levels of activity. Synthetic water solutions A1-A3 containing pure alpha emitters, B1-B3 containing pure beta emitters and AB1-AB7 containing alpha and beta emitters were analysed following Procedure 1. Three replicates of each analysis were performed.

Accuracy and repeatability were evaluated at the different activity ratios established from the bias and the relative standard deviation values were obtained. The results are shown in Table 2.

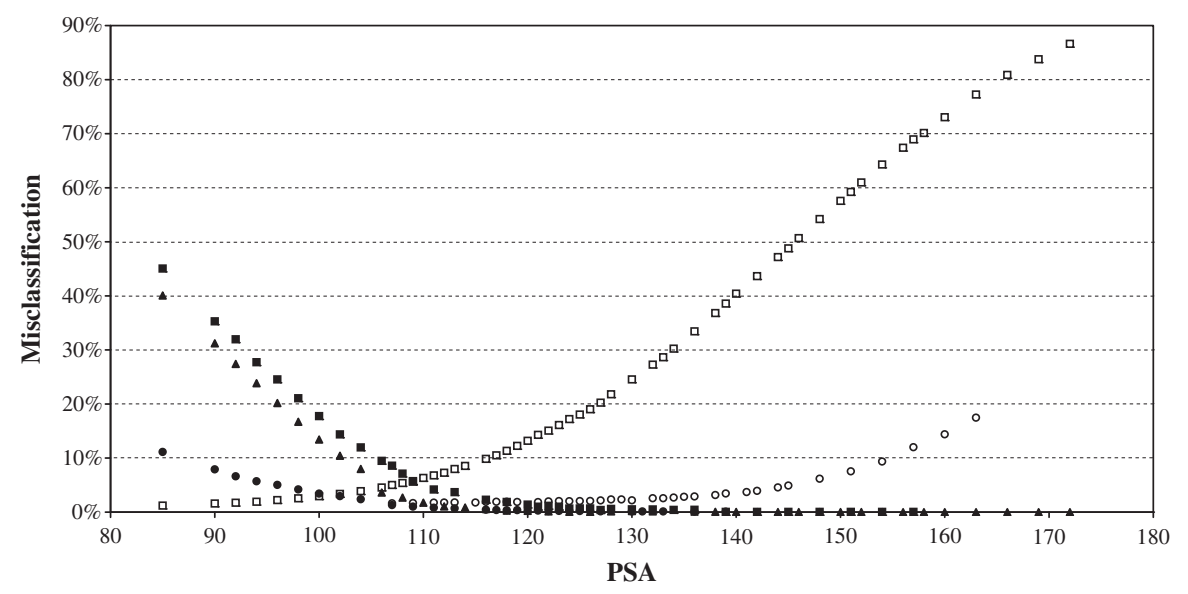

Fig. 1. Alpha and beta misclassification percentages as a function of the PSA value for different alpha and beta emitters ( ${ }^{137} \mathrm{Cs}, \boldsymbol{\square}{ }^{40} \mathrm{~K}, \boldsymbol{\Delta}{ }^{90} \mathrm{Y}$, $\square{ }^{236} \mathrm{U}$ and $\left.\circ{ }^{241} \mathrm{Am}\right)$. 
The LRA acceptance criteria are a maximum bias of 50\% for gross alpha determination and 30\% for gross beta determination. Maximum relative standard deviation values were established on the basis of the activity concentration: $30 \%$ standard deviation values were accepted for activity levels up to ten times the MDA, 25\% for activity levels up to 100 times the MDA and $15 \%$ for higher activity concentrations. The results obtained for pure alpha and beta solutions (A1-A3, B1-B3) show adequate accuracy and repeatability values according to these criteria, except for the B1 solution, where the bias exceeded $30 \%$.

In the case of mixed radionuclide samples, the results were within the acceptance range when the activities were less than tenfold more than the MDA. In the case of low activity concentrations, the bias for the alpha activity at the MDA level was over $100 \%$ when the beta level was one hundredfold higher than the alpha level (AB2, AB3). In the case of the beta measurements, the bias was also over $100 \%$ at the MDA level when the alpha activity level was an order of magnitude higher than the beta activity (AB6). Considering the different levels of activity in these solutions, the results showed the effect that the remaining misclassification had on the determination of gross alpha/beta activities. However, the results for pure and mixed radionuclide samples at any one activity level also show great variability. For example, for solutions A1, B1 and AB1, the bias in the mixed sample was reduced approximately $30 \%$ from pure emitter solutions.

The high bias values obtained at some levels of activity concentration were a serious drawback to the application of this method for real samples, where the actual content is not known.

Table 2

The accuracy and repeatability values for Procedure $1\left(\mathrm{HNO}_{3} \mathrm{pH} 1.5\right)$.

\begin{tabular}{|c|c|c|c|c|c|c|c|c|c|}
\hline & \multicolumn{2}{|c|}{ Accuracy } & \multicolumn{2}{|c|}{ Repeatability } & & \multicolumn{2}{|c|}{ Accuracy } & \multicolumn{2}{|c|}{ Repeatability } \\
\hline & $\begin{array}{l}\text { Alpha } \\
(\%)\end{array}$ & $\begin{array}{l}\text { Beta } \\
(\%)\end{array}$ & $\begin{array}{l}\text { Alpha } \\
(\%)\end{array}$ & $\begin{array}{l}\text { Beta } \\
(\%)\end{array}$ & & $\begin{array}{l}\text { Alpha } \\
(\%)\end{array}$ & $\begin{array}{l}\text { Beta } \\
(\%)\end{array}$ & $\begin{array}{l}\text { Alpha } \\
(\%)\end{array}$ & $\begin{array}{l}\text { Beta } \\
(\%)\end{array}$ \\
\hline A1 & 36.5 & - & 18.6 & - & AB1 & 7.8 & -4.6 & 9.5 & 2.8 \\
\hline A2 & 7.4 & - & 4.0 & - & $\mathrm{AB} 2$ & 104.7 & -2.1 & 7.3 & 2.1 \\
\hline A3 & 7.3 & - & 1.7 & - & AB3 & 923.0 & -0.4 & 2.2 & 1.0 \\
\hline B1 & - & -37.9 & - & 8.6 & AB4 & -2.2 & 33.7 & 1.3 & 8.3 \\
\hline B2 & - & 2.2 & - & 5.1 & AB5 & 1.1 & 3.4 & 1.4 & 0.3 \\
\hline \multirow[t]{2}{*}{ B3 } & - & 2.3 & - & 0.4 & AB6 & -5.2 & 111.7 & 0.3 & 5.1 \\
\hline & & & & & AB7 & 1.8 & 2.7 & 0.7 & 1.8 \\
\hline
\end{tabular}

\section{3. $p H$ and acid effects}

\subsubsection{The effect of $p H$}

The method established for gross alpha/beta determination showed great variability in the results despite the simple procedure. Because the only reagent used was the acid, which was added before preconcentration of the sample, the effect of the final $\mathrm{pH}$ was studied.

Fig. 2 shows the variation of alpha and beta misclassification against $\mathrm{pH}$ change in $\mathrm{HNO}_{3}$ media. In the $\mathrm{pH}$ range studied (0.5-3.0), alpha misclassification diminished from $60 \%$ to $7 \%$. In the region below a $\mathrm{pH}$ of 2 , misclassification was strongly affected by small variations in $\mathrm{pH}$. In the case of beta misclassification, the effect of $\mathrm{pH}$ is less significant, increasing only slightly from $1 \%$ to $4 \%$.

Considering that when using Procedure 1 , the final $\mathrm{pH}$ would be between 1 and 2, a variation of 15\% in the misclassification of samples would be expected. This variability would also contribute to the high bias described in Section 3.2.

As for the quench parameter, no differences were observed in the SQP[E] values or the shape of the external source spectra as the $\mathrm{pH}$ decreased. Only $\mathrm{pH} 0.5$ media produced a significant reduction of the quench parameter value and variation in the shape of the external source spectra.

\subsubsection{The effect of the acid}

Two possibilities were considered in order to optimise the final conditions for analysis: working at higher $\mathrm{pH}$ values using $\mathrm{HNO}_{3}$ and looking for a different acid, which did not produce problems at lower $\mathrm{pH} . \mathrm{HCl}$ is a strong mineral acid that is non-oxidising and produces low $\mathrm{pH}$ values without any interfering oxidation-reduction reactions, such as those caused by $\mathrm{HNO}_{3}$. The curves of alpha and beta misclassifications for ${ }^{236} \mathrm{U}$ and ${ }^{90} \mathrm{Sr} /{ }^{90} \mathrm{Y}$ at $\mathrm{pH} 1.5$ and 2.5 using $\mathrm{HNO}_{3}$ and $\mathrm{HCl}$ are presented in Fig. 3. These results show the different behaviour of the alpha and beta misclassifications as the acid changed. $\mathrm{HNO}_{3}$ at $\mathrm{pH} 1.5$ produced higher alpha misclassification than the other three combinations, which show similar values. When $\mathrm{HCl}$ was used, alpha misclassification was not affected by the final $\mathrm{pH}$ value. In the case of beta misclassifications, the data were aligned into two groups corresponding to the two $\mathrm{pH}$ values analysed. In this case, higher $\mathrm{pH}$ values produced higher misclassification. These two plots tended to converge when PSA values were over 100. The optimum PSA for the different media tested was 110 , with the $\mathrm{HNO}_{3}$ medium at $\mathrm{pH}$ 1.5 producing higher misclassification values.

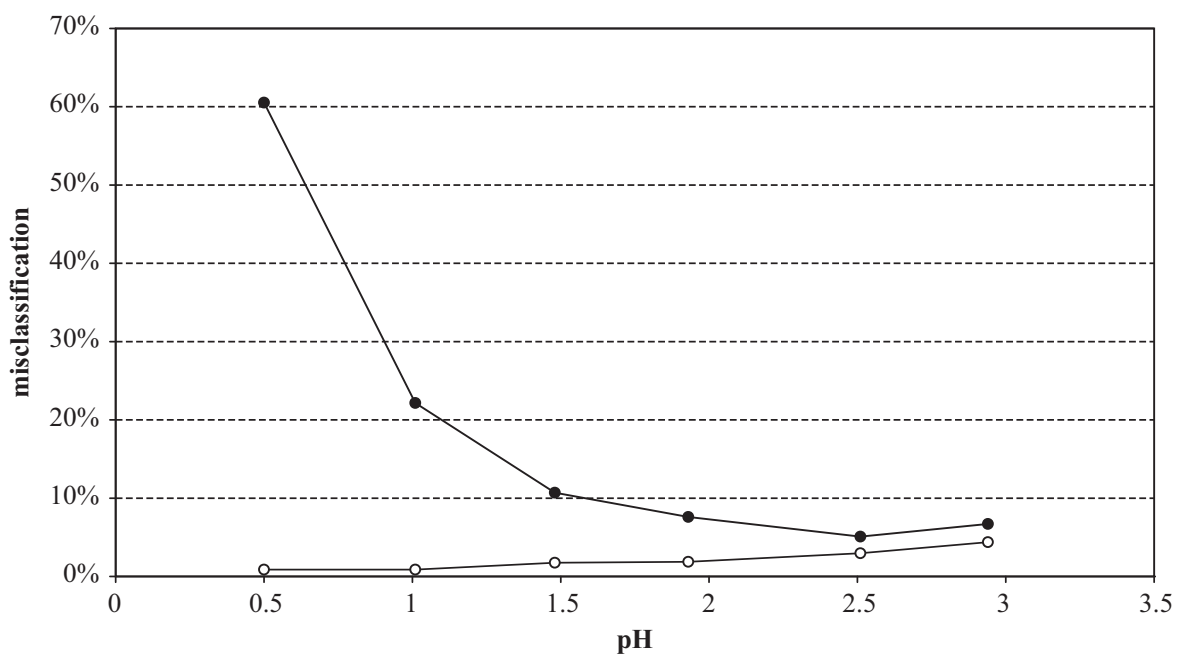

Fig. 2. Alpha ( $)$ and beta $(\circ)$ misclassification percentages as a function of pH using $\mathrm{HNO}_{3}$. 


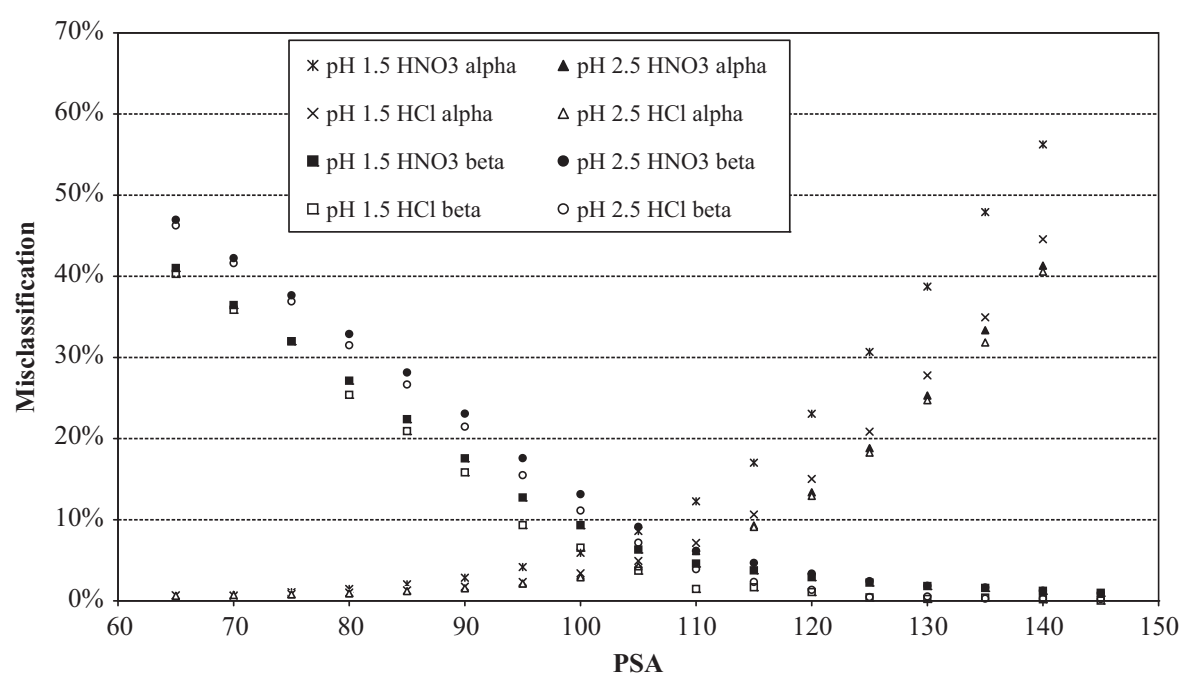

Fig. 3. Alpha $\left({ }^{236} \mathrm{U}\right)$ and beta $\left({ }^{90} \mathrm{Sr} /{ }^{90} \mathrm{Y}\right)$ misclassification percentages as a function of $\mathrm{PSA}$ for $\mathrm{HNO}_{3}$ and $\mathrm{HCl}$ media at two pH values.

Table 3

The minimum detectable activities (MDA) of the different procedures applied to determine gross alpha and gross beta activity.

\begin{tabular}{lll}
\hline & Alpha MDA $\left(\mathrm{Bq} \mathrm{l}^{-1}\right)$ & Beta MDA $\left(\mathrm{Bq} \mathrm{l}^{-1}\right)$ \\
\hline Procedure 1 & 0.028 & 0.160 \\
Procedure 2 & 0.033 & 0.149 \\
Procedure 3 & 0.025 & 0.144 \\
\hline
\end{tabular}

No differences were observed in the quench parameter value or the shape of the external source spectra for this part of the study.

According to the results of this study, the final media were modified to keep misclassification at low values. The most suitable conditions were $\mathrm{pH} 2.5$ when using $\mathrm{HNO}_{3}$ and $\mathrm{pH} 1.5$ when using $\mathrm{HCl}$.

\subsection{The evaluation of the modified method's performance}

Procedures 2 and 3 were modifications of Procedure 1 that used the optimised media: $\mathrm{HNO}_{3}$ at $\mathrm{pH} 2.5$ (Procedure 2) and $\mathrm{HCl}$ at pH 1.5 (Procedure 3). A series of blanks were analysed, and the MDA was calculated for the two procedures. The MDA values for both procedures are presented in Table 3 and were similar to those from Procedure 1.

Evaluations of the accuracy and repeatability of the procedures were carried out. Seven different solutions $\left(A B 1^{*}-A B 7^{*}\right)$ with different concentrations of alpha and beta emitters where analysed using Procedures 2 and 3. Three replicates of each assay were carried out. The results in Table 4 show that the remaining alpha misclassifications in the beta determination were removed in both cases, with relative errors below $15 \%$ for all the samples and repeatability values below $10 \%$.

In the case of gross alpha determination, the results showed that the bias at a fixed alpha concentration increased as the concentration of beta emitters increased. This effect was stronger when the concentration of alpha emitters was near the MDA level (AB1*$\left.A B 3^{*}\right)$, and the bias remained over $100 \%$ at the maximum concentration of beta emitters $\left(\mathrm{AB} 3^{*}\right)$. The beta concentration also affected the gross alpha determination at higher levels of alpha concentration, but when alpha concentrations were tenfold higher than the MDA $\left(A B 4^{*}-\mathrm{AB}^{*}\right)$, the trueness values were below $30 \%$, which was adequate for such determinations.
Table 4

The accuracy and repeatability values for Procedures 2 and 3.

\begin{tabular}{|c|c|c|c|c|c|c|c|c|}
\hline & \multicolumn{4}{|c|}{ Procedure $2\left(\mathrm{HNO}_{3} \mathrm{pH} 2.5\right)$} & \multicolumn{4}{|c|}{ Procedure $3(\mathrm{HCl} \mathrm{pH} 1.5)$} \\
\hline & \multicolumn{2}{|c|}{ Accuracy } & \multicolumn{2}{|c|}{ Repeatability } & \multicolumn{2}{|c|}{ Accuracy } & \multicolumn{2}{|c|}{ Repeatability } \\
\hline & $\begin{array}{l}\text { Alpha } \\
(\%)\end{array}$ & $\begin{array}{l}\text { Beta } \\
(\%)\end{array}$ & $\begin{array}{l}\text { Alpha } \\
(\%)\end{array}$ & $\begin{array}{l}\text { Beta } \\
(\%)\end{array}$ & $\begin{array}{l}\text { Alpha } \\
(\%)\end{array}$ & $\begin{array}{l}\text { Beta } \\
(\%)\end{array}$ & $\begin{array}{l}\text { Alpha } \\
(\%)\end{array}$ & $\begin{array}{l}\text { Beta } \\
(\%)\end{array}$ \\
\hline $\mathrm{AB} 1^{*}$ & 24.5 & 6.5 & 0.2 & 5.7 & 15.8 & -4.2 & 15.3 & 1.9 \\
\hline $\mathrm{AB} 2^{*}$ & 70.4 & 2.6 & 6.9 & 0.4 & 49.0 & 0.5 & 3.7 & 0.8 \\
\hline $\mathrm{AB} 3^{*}$ & 681.2 & 2.7 & 9.8 & 1.1 & 414.8 & 0.7 & 12.3 & 1.9 \\
\hline $\mathrm{AB} 4^{*}$ & 5.8 & 1.8 & 2.6 & 4.6 & -0.3 & -3.9 & 7.0 & 7.4 \\
\hline $\mathrm{AB} 5^{*}$ & 11.7 & 1.8 & 2.5 & 4.1 & 0.2 & 3.9 & 1.3 & 3.7 \\
\hline $\mathrm{AB} 6^{*}$ & -0.1 & 9.6 & 1.4 & 3.3 & 0.2 & 11.9 & 0.8 & 3.1 \\
\hline $\mathrm{AB} 7^{*}$ & 26.6 & 3.5 & 2.3 & 3.6 & 13.9 & 2.7 & 1.3 & 0.3 \\
\hline
\end{tabular}

Repeatability values were below $10 \%$ in most cases, with a maximum of $15 \%$ for some alpha determinations at the MDA level, which were acceptable values according to LRA criteria.

These results showed that $\mathrm{HCl}$ at $\mathrm{pH} 1.5$ was the most suitable media for measuring gross alpha and gross beta activities using liquid scintillation, although neither of the two procedures gave an acceptable bias for the gross alpha determination of solution AB3*. Fig. 4 shows the alpha and beta spectra obtained for sample AB3* $^{*}$ after Procedure 3 was applied. Detailed analysis of the spectra allowed additional considerations to be made. The vertical lines represent the upper and lower limits of the gross alpha counting window. The beta spectrum had the expected shape of a two-emitter spectrum, although the ${ }^{90} \mathrm{Y}$ band had lower intensity than the ${ }^{90} \mathrm{Sr}$ band, despite being in secular equilibrium. In the alpha spectrum, the signal corresponding to ${ }^{236} U$ (channels 600-650) appeared with a second peak in the higher regions of the spectrum (channels 750-925). It is clear from the figure that this peak was produced by the ${ }^{90} \mathrm{Y}$ signal, which was misclassified by the counter as evidenced by the reduction in intensity of the beta spectrum. Despite the fact that misclassification of the ${ }^{90} \mathrm{Y}$ signal into the alpha spectra was high, most of the misclassification appeared in channels over the upper limit of the gross alpha window. No significant misclassification was observed in the ${ }^{90} \mathrm{Sr}$ region. Considering that beta emitters present in the environment have emission energies much lower than ${ }^{90} \mathrm{Y}$, misclassification would normally be lower, even for samples that contained high concentrations of beta emitters. 


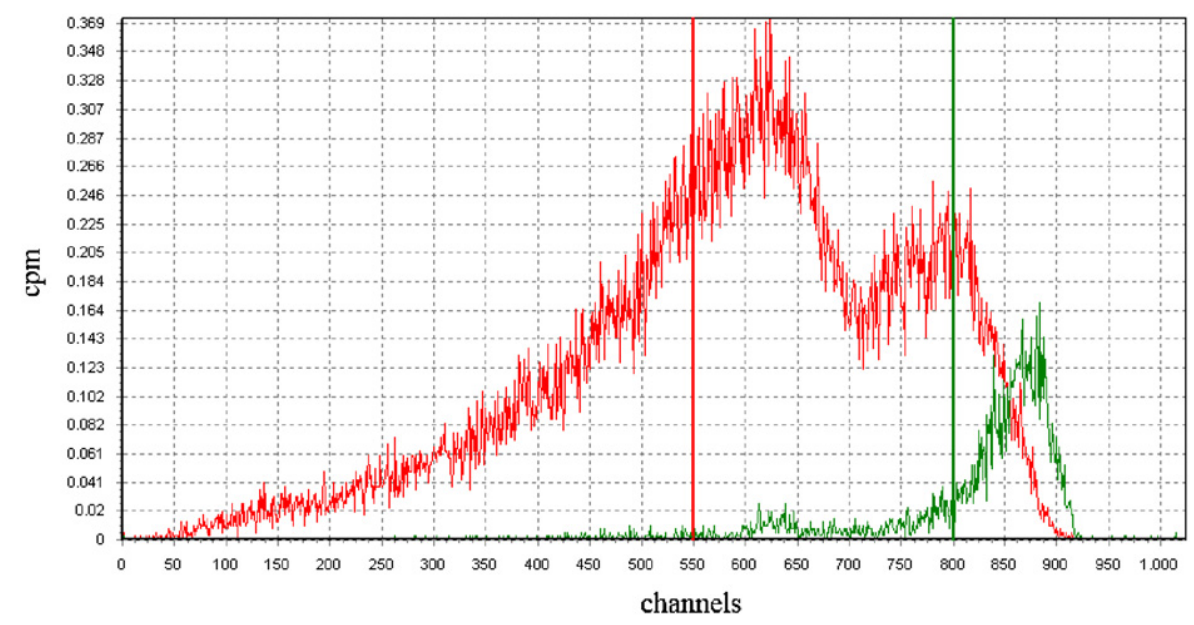

Fig. 4. The gross alpha and gross beta spectra of $A B 3^{*}$ using Procedure 3.

Table 5

The results from real samples analysed by applying Procedure 3.

\begin{tabular}{|c|c|c|c|c|c|c|c|c|}
\hline & \multicolumn{4}{|c|}{ Gross alpha } & \multicolumn{4}{|c|}{ Gross beta } \\
\hline & \multicolumn{2}{|c|}{ Reference } & \multicolumn{2}{|c|}{ LRA result } & \multicolumn{2}{|c|}{ Reference } & \multicolumn{2}{|c|}{ LRA result } \\
\hline & $\begin{array}{l}A_{\alpha} \\
\left(\mathrm{Bq} \mathrm{l}^{-1}\right)\end{array}$ & $\begin{array}{l}u_{c} \\
(k=2)\end{array}$ & $\begin{array}{l}A_{\alpha} \\
\left(\mathrm{Bq} \mathrm{l}^{-1}\right)\end{array}$ & $\begin{array}{l}u_{c} \\
(k=2)\end{array}$ & $\begin{array}{l}A_{\beta} \\
\left(\mathrm{Bq} \mathrm{l}^{-1}\right)\end{array}$ & $\begin{array}{l}u_{c} \\
(k=2)\end{array}$ & $\begin{array}{l}A_{\beta} \\
\left(\mathrm{Bq} \mathrm{l}^{-1}\right)\end{array}$ & $\begin{array}{l}u_{c} \\
(k=2)\end{array}$ \\
\hline S1 & 0.071 & 0.017 & 0.076 & 0.017 & 0.15 & 0.07 & 0.14 & 0.09 \\
\hline S2 & 0.68 & 0.02 & 0.39 & 0.03 & 3.09 & 0.14 & 3.15 & 0.12 \\
\hline S3 & 1.92 & 0.04 & 1.37 & 0.06 & 4.39 & 0.16 & 4.64 & 0.14 \\
\hline S4 & 2.07 & 0.05 & 2.16 & 0.07 & 1.10 & 0.12 & 1.16 & 0.10 \\
\hline S5 & 18.8 & 1.6 & 21.9 & 0.2 & 51 & 4 & 61.0 & 0.4 \\
\hline
\end{tabular}

\subsection{The analysis of real samples}

A series of previous performance test samples were analysed in the final part of the study to address the application of Procedure 3 to real samples. All samples had been analysed in previous intercomparison exercises and included natural surface and groundwater (S1 and S4) as well as synthetic samples (S2, S3, S5). No precipitation or colloid formation was observed due to the change of acid, and the spectra from all samples showed adequate discrimination of the alpha and beta signals. These results are presented in Table 5 and provide evidence that the proposed method performs well with different types of samples and levels of activity.

\section{Conclusions}

The effect that alpha and beta energies have on PSA optimisation for the simultaneous determination of gross alpha/beta activities was studied. The optimum PSA was not affected by the energy of the beta emitter chosen for the calibration. For alpha particles, the optimum PSA shifted to higher values as the energy increased. For that reason, while most beta emitters in the intermediate-to-high beta energy range were adequate for instrument optimisation, the alpha emitters chosen should be in the lower region of the alpha energy range. Optimisation with a lowenergy alpha emitter did not increase misclassification when alpha emitters of higher energy were measured. Calibration with high-energy alpha emitters could be used for specific applications, such as specific radionuclide determinations or in accident situations, but should not be used for routine gross alpha/beta determinations in surveillance programs and environmental control.

The effect of $\mathrm{pH}$ on the simultaneous determination of gross alpha/beta activities by liquid scintillation counting was addressed using two common strong mineral acids $\left(\mathrm{HNO}_{3}\right.$ and $\left.\mathrm{HCl}\right)$. In spite of the many procedures available using $\mathrm{HNO}_{3}$, alpha misclassification increased strongly when $\mathrm{HNO}_{3}$ was used at a $\mathrm{pH}$ below 2. The external quench parameter did not show significant variation when the $\mathrm{pH}$ was changed. $\mathrm{HCl}$ was a better option because the final $\mathrm{pH}$ could be adjusted to approximately 1 , and the total misclassification was maintained below $10 \%$. If $\mathrm{HNO}_{3}$ is required for the analysis, the final pH should not be below 2.5 in order to assure that alpha misclassifications are maintained at approximately $5 \%$. Beta misclassification was less affected by $\mathrm{pH}$ variation, which is a behaviour observed for both of the two acids studied. All procedures achieved MDA values one order of magnitude below the threshold level legislated for drinking water in Spain, which makes them adequate for such analyses.

Misclassification in the simultaneous measurement of gross alpha/beta activities could not be completely removed, and the effect of the remaining misclassification was studied at different levels of activity concentration. Increasing final $\mathrm{pH}$ value to 2.5 or changing the acid to $\mathrm{HCl}$ removed the effect on beta activity determination caused by alpha emitters present in the sample. However, remaining beta misclassification could not be completely removed, and relative errors at the MDA level exceeded 30\% when the gross beta activity was two orders of magnitude higher than the alpha activity. This misclassification is considered the maximum expected error because it was calculated using ${ }^{90} \mathrm{Y}$, and most other commonly found beta emitters have lower energies, including those in most environmental and potable water samples. Moreover, in the case of a contamination episode in which artificial radionuclides such as ${ }^{90} \mathrm{Y}$ would be expected, the quantity of alpha emitters would probably not remain at the MDA level.

The application of the modified procedure using $\mathrm{HCl}$ performed well during the analysis of real samples. However, in the case of samples that contain artificial beta radionuclides or very small alpha/beta ratios, the possibility of incorrect quantification of the gross alpha concentration due to remaining misclassification and different activity ratios needs to be considered.

\section{Acknowledgements}

This work was developed with the support of the "Commissionat per a Universitats $i$ Recerca" of the "Departament d'Innovació, 
Universitats i Empresa" of the "Generalitat de Catalunya" and the European Social Fund. This work was partially funded by the Spanish Government (CICYT, contract CTM2005-03847) and the Spanish Nuclear Safety Council (project "Study of existing problems in the determination of the total alpha activity in drinking water. Proposal for procedures.").

\section{References}

Currie, L.A., 1968. Limits for qualitative detection and quantitative determination. Application to radiochemistry. Anal. Chem. 40, 586-593.

Dávila Rangel, J., López del Río, H., Rodríguez, B., Solache-Ríos, B., 2001. Gross alpha and gross beta radioactivity in drinking water from Zacatecas and Guadalupe cities Mexico. J. Radioanal. Nucl. Chem. 247, 425-428.

DeVol, T.A., Theisen, C.D., DiPrete, D.P., 2007. Effect of quench on alpha/beta pulse shape discrimination of liquid scintillation cocktails. Health Phys. 92, S105-S111. European Council, 1998. Directive 98/83/CE of 3rd November 1998 on the quality of water intended for human consumption. Off. J. Eur. Union L330/332-354.

Forte, M., Rusconi, R., Cazzaniga, M.T., Sgorbati, G., 2007. The measurement of radioactivity in Italian drinking waters. Microchem. J. 85, 98-102.

ISO, International Organization for Standardization, 2010. ISO 11704:2010. Water quality. Measurement of gross alpha and beta activity concentration in nonsaline water - Liquid scintillation counting method ISO, Suïssa.

Kleinschmidt, R.I., 2004. Gross alpha and beta activity analysis in water - a routine laboratory method using liquid scintillation analysis. Appl. Radiat. Isot. 61, 333-338.

Mas, J.L., García-León, M., García-Tenorio, R., Bolívar, J.P., 2007. Radionuclide concentrations in water. In: Pöschl, M., Nollet, L.M.L. (Eds.), Environmental Concentrations in Food and the Environment. CRC Press, Boca Raton, pp. 59-111.

Palomo, M., Villa, M., Casacuberta, N., Peñalver, A., Borrull, F., Aguilar, C., 2011. Evaluation of different parameters affecting the liquid scintillation spectrometry measurement of gross alpha and beta index in water samples. Appl. Radiat. Isot. 69, 1274-1281.

Pates, J.M., Cook, G.T., MacKenzie, A.B., Pass, C.J., 1998. Implications of beta energy and quench level for alpha/beta liquid scintillation spectrometry calibration. Analyst 123, 2201-2207.

Pujol, L., Sanchez-Cabeza, J.-A., 1997. Role of quenching on alpha/beta separation in liquid scintillation counting for several high capacity cocktails. Analyst 122 383-385 (Cambridge, United Kingdom).
Rodríguez Barquero, L., Grau Carles, A., 1998. The influence of the primary solute on alpha/beta discrimination. Appl. Radiat. Isot. 49, 1065-1068.

Ruberu, S.R., Liu, Y.-G., Perera, S.K., 2008. An improved liquid scintillation counting method for the determination of gross alpha activity in groundwater wells. Health Phys. 95, 397-406.

Rusconi, R., Forte, M., Caresana, M., Bellinzona, S., Cazzaniga, M.T., Sgorbati, G., 2006. The evaluation of uncertainty in low-level LSC measurements of water samples. Appl. Radiat. Isot. 64, 1124-1129.

Salonen, L., 2006a. Alpha spillover depends on alpha energy: a new finding in alpha/beta liquid scintillation spectrometry. LSC 2005, Advances in Liquid Scintillation Spectrometry, International Conference, Katowice, Poland, 2005, pp. 135-148.

Salonen, L., 2006b. Alpha/beta liquid scintillation spectrometry in surveying Finnish groundwater samples. Radiochemistry 48, 606-612.

Spain, 2003. Royal Decree 140/2003, of 7th February on the health criteria of water quality for human consumption (Real Decreto 140/2003, de 7 de febrero, por el que se establecen los criterios sanitarios de la calidad del agua de consumo humano), in: BOE (Ed.), 45, Madrid, pp. 7228-7245.

USEPA, United States Environmental Protection Agency, 2000. National Primary Drinking Water Regulations; Radionuclides; Final Rule EPA, Federal Register 65:236, 76708-76753.

Villa, M., Manjón, G., Garcia-Leon, M., 2003. Study of colour quenching effects in the calibration of liquid scintillation counters: the case of Pb-210. Nucl. Instrum. Methods Phys. Res., Sect. A 496, 413-424.

WHO, World Health Organisation, 2008. Guidelines for drinking-water quality incorporating 1st and 2nd addenda, Vol.1 Recommendations, 3rd ed. WHO, Geneva.

Wong, C.T., Soliman, V.M., Perera, S.K., 2005. Gross alpha/beta analyses in water by liquid scintillation counting. J. Radioanal. Nucl. Chem. (264), 357-363.

Yang, D., 1996. Calibration and quench correction for alpha liquid scintillation analysis. In: Cook, G.T., Harkness, D.D., MacKenzie, A.B., Miller, B.F., Scott, E.M. (Eds.), Liquid Scintillation Spectrom., 1994; 1996, pp. 339-344.

Zapata-García, D., Llauradó, M., 2009. Study of instrumental parameters affecting the simultaneous measurement of gross alpha and gross beta activities in water samples. In: Eikenberg, J., Maya, J., Beer, H., Baehrle, H. (Eds.), LSC 2008, Advances in Liquid Scintillation Spectrometry, pp. 59-69.

Zapata-García, D., Llauradó, M., Rauret, G., 2009. Establishment of a method for the rapid measurement of gross alpha and gross beta activities in sea water. Appl. Radiat. Isot. 67, 978-981. 